\title{
PHOTOISOMERIZATON
}

\section{Molecular motors driven by light}

The demonstration of light-powered unidirectional molecular motion suggests that a new breed of artificial nanoscale motors and pumps could be a reality in the near future. Giulio Ragazzon and co-workers at the Photochemical Nanosciences Laboratory at the University of Bologna in Italy report how a molecular 'axle' can be made to move through a macrocycle 'ring' following illumination with ultraviolet light (Nature Nanotech. http://dx.doi.org/10.1038/nnano.2014.260; 2014). The axle is composed of three parts: a photoswitchable $E$-azobenzene unit at one end (green/red structure in image), a central ammonium recognition site (purple) and a passive methylcylopentyl pseudo-stopper at the other end (white sphere). The macrocycle ring (pink) is composed of 2,3-dinaphtho[24]crown-8 ether $\mathbf{1}^{24}$ With the appropriate illumination, a photoisomerization reaction is triggered and the photoswitchable end of the axle changes from an $E-2^{+}$configuration (green) to a bent $Z-\mathbf{2}^{+}$state (red) and

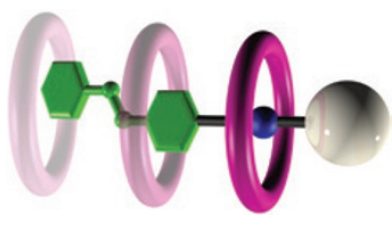

Light

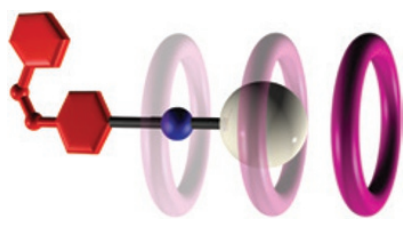

the axle moves relative to the ring. For irradiation with $365 \mathrm{~nm}$ light, the $E$ to $Z$ state conversion is estimated at $96 \%$ and experimental tests indicate that almost complete conversion occurs within an illumination period of five minutes. As the $E-2^{+}$and $Z-2^{+}$states of the axle have different absorption and luminescence spectra, the motion can be observed by monitoring the time-dependent changes in emission. The authors report that the effect is reversible as the same light can also trigger a state-change back to the initial $E-2^{+}$state because the $E$ - and Z-azobenzene have overlapping absorption spectra. Simulations suggest that around 430 photons of $365 \mathrm{~nm}$ light are needed to complete a motion cycle. The cycle is expected to be highly resistant to fatigue and by using a series of periodic flashes the authors say that it should be possible to produce directional and repetitive movements. In the future it is postulated that the lightdriven assembly could be used to create miniature molecular pumps capable of generating concentration gradients across membranes. The team is now investigating the design of more complex three-dimensional assemblies.

\section{Expanding ambitions}

Long-distance secure quantum communication has long been one of the goals of quantum optics research. Ambitions are now growing following the realization of fibre-based links to free-space satellite-ground communication networks.

\section{Noriaki Horiuchi}

( uantum mechanics promises a way of overcoming technological limitations in many areas of science. Although many quantum schemes are still laboratorybased and in their infancy - such as quantum-enhanced metrology and quantum computing - secure communication through quantum key distribution (QKD) is rapidly becoming a practical reality. Progress in the area was certainly one of the hot topics of discussion at the Conference on Quantum Communication, Measurement and Computing (QCMC) held in Hefei, China, over 2-6 November 2014.

Eleni Diamanti of Télécom ParisTech in France explained that the current technological limit of long-distance secure communication based on QKD is imposed by the amount of excess noise in the link. In her talk, she discussed how the imperfections and limitations of practical systems impact the security of continuousvariable quantum communication. To further extend the distance of secure communication, she has developed novel error correction algorithms operating at very low signal-to-noise ratios and she has also built an optical system capable of functioning stably in these conditions. She told Nature Photonics that she is now trying to fabricate a QKD system on a chip. "It will be interesting to examine the difference between the bulk and the chip QKD systems and to compare their limitations," she said.

In principle, quantum amplification can help increase the length of a fibrebased quantum communication system and is implemented by the intentional introduction of a number of regenerative relays or 'repeaters' at intermediate points along the transmission channel. However, the characteristics of the repeaters are critical. Norbert Lütkenhaus of the University of Waterloo in Canada theoretically investigated whether Gaussian-operation repeaters could serve to increase the length of longdistance QKD. His investigation into the 